\title{
Electrostatic charging of wind-blown dust and implications on dust transport
}

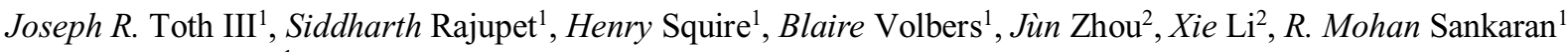 \\ and Daniel J. Lacks ${ }^{1}$ \\ ${ }^{1}$ Case Western Reserve University, Department of Chemical and Biomolecular Engineering, Cleveland, Ohio 44106, USA \\ ${ }^{2}$ Key Laboratory of Mechanics on Disaster and Environments in Western China, Ministry of Education of China, Lanzhou \\ University, Lanzhou, Gansu 730000, China
}

\begin{abstract}
It is well known that electric fields occur in wind-blown dust, due to the triboelectric charging of particles as they collide. Triboelectric charging, or contact electrification, is a poorly understood and complex phenomenon. It is especially important in granular systems, as the high surface-to-volume ratio can lead to the build-up of large amounts of charge. A particularly surprising effect, which is important in dust systems, is that charge transfer occurs in systems of a single composition, such that there is a particle-size dependent polarity of the particles. Here, we use a combined experimental and theoretical approach to elucidate the electrostatic charging that occurs during dust storms, and the effects of this electrostatic charging on dust transport. We create laboratory-scale wind-blown dust systems, and study the electrostatic charging. We find that larger particles tend to charge positive and to stay at or near the sand bed, while smaller particles tend to charge negative and get lofted to higher elevations. This self-segregating of charged particles would lead to electric fields within a dust storm. Our results show that electric fields then increase the dust transport by more easily lofting charged particles.
\end{abstract}

\section{Introduction}

For over a century, scientists have known that large electric fields develop in dust storms [1]. The electric fields come about from the electrostatic charging of the dust particles. The electrostatic charging effects can be very dramatic, as described in a book about America's "Dust Bowl" in the 1930's, when catastrophic dust storms ravaged parts of the country [2]:

"As the black wall approached, car radios clicked off, overwhelmed by the static. Ignitions shorted out...Every car dragged a metal chain to ground the static...Men avoided shaking hands with each other because the static electricity was so great it could knock a person down."

More importantly, electrostatic charge on the dust particles is believed to significantly increase the magnitude of wind-blown dust $[3,4]$. The electrostatic effects must be understood if dust transport processes - which affect many aspects of the climate - are to be accurately modelled. Of particular relevance is the role of wind-blown dust in desertification, a serious problem in Asia and Africa.
Dust particles become electrostatically charged in wind-blown systems by triboelectric charging, which is the process where surfaces become electrically charged by contacts with one another. We encounter triboelectric charging almost every day of our lives. For example, we often get a shock touching a door-knob after walking across a carpet. Even children are familiar with rubbing a balloon on their hair, which makes their hair stand on end.

In granular systems, the high ratio of surface area to volume results in large amounts of charge being exchanged. For this reason, the particles in dust storms can become electrically charged $[5,6]$.

But the electrostatic charging in dust storms presents an interesting question - the dust particles are composed of the same material, so why would triboelectric charging occur when two dust particles collide? Surprisingly, even materials of identical chemical composition have a tendency to exchange electric charge [7]. In systems where the surface area of one object is different from that of another object, the direction of charging is found to be such that the larger-surface-area object loses more charge, and the smaller-surface-area object gains more charge. In granular systems, this effect has been shown to lead 
to large particles charging positively, and small particles charging negatively [8]. This phenomenon comes into play in dust storms, where the smaller particles are lofted higher and have a more negative charge, and the larger particles remain lower and have a more positive charge. This charge separation - negative charges higher and positive charges lower - gives rise to the electric fields in dust storms.

Here we describe experiments used to investigate the triboelectric charging of particles in wind-blown dust $[9,10]$. One system mimics a sandstorm with wind blowing the across a bed and then between two electrodes. These experiments give details of the movements of dust particles due to electric fields in a dust storm, as well as the charge distribution in relation to the height above the ground. A second system studies particle charging in a more controlled manner, and enables a clear elucidation of the size dependence of particle charging under different conditions.

We coupled a Monte Carlo simulation model with these experiments, to understand the reasons for triboelectric charging and the particle size dependence. Our model is based on a theory we proposed [11], in the contact of one surface with another provides a path for an electron or ion to relax from a higher energy state on one particle to a lower energy state on the other particle.

\section{Methods}

To mimic a sand storm scenario, where blowing sand undergoes triboelectric charging, an experimental system with a bed of sand and two horizontal electrodes was used, as shown in Figure 1. The electrodes are oriented horizontally to create an electric field is in the vertical direction; the electric field and gravity both influence the movements of the particles in the direction perpendicular to the ground. Sand particles become triboelectrically charged by saltation across the sand bed. The charge of the particles is measured with Faraday cups attached to electrometers, and the size distribution of particles is determined by sieving the collected particles.

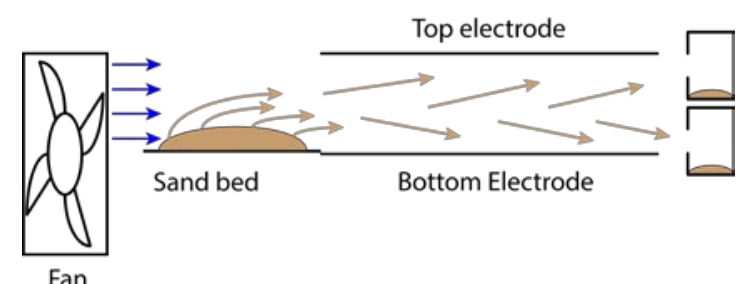

Fig. 1. Schematic of a simulated sand storm system. Air blows across the sand bed, picking up sand and passing it through the electrodes and drops it into the faraday cups.

To carry out more controlled experiments on the triboelectric charging in granular systems, we use a different experimental setup. To isolate the triboelectric charging effects arising solely from particle to particle interactions, a system was designed using a particle fountain and an electrostatic separator, as shown in Figure 2 [9,10]. An air stream passing up through a bed of particles forms a fountain where particles are colliding with other particles but not any other surfaces. After the particles are charged, a separate air stream blows across the top of the fountain, pushing the particles over an electrostatic separator. The separator consists of two electrodes with a $12 \mathrm{kV}$ difference between them. The particles fall through the separator, deflect towards the electrode with the opposite polarity, and are collected at the bottom into various collection cups. The particles in each cup are sieved to determine the size distribution. This system was operated inside a humidity controlled container, allowing experiments to be carried out at chosen values of relative humidity.

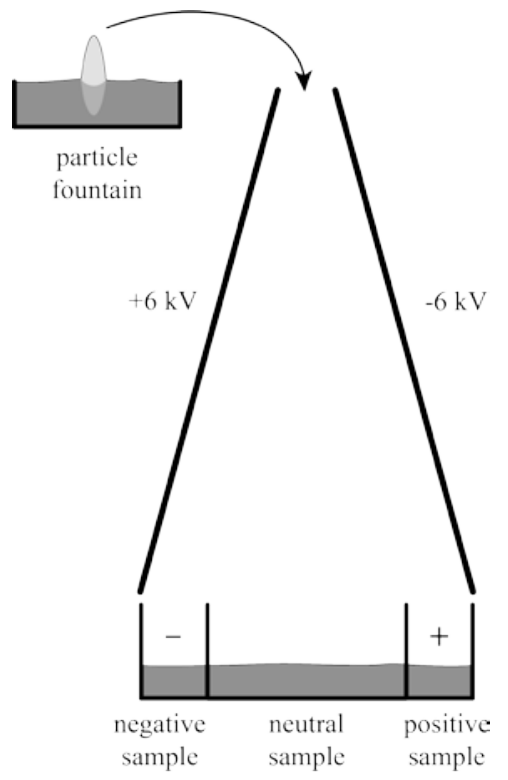

Fig. 2. Schematic of particle fountain and electrostatic separator consisting of two electrodes. Particles from the fountain pass down through the electrodes into the different collection boxes at the bottom.

Monte Carlo simulations were used to understand the physical basis of the experimental results. The model considers a collection of particles with a bidisperse size distribution. On the particles are charge carriers (these could be electrons or ions), which could be in either high or low energy states. We simulate the effects of a flow of these particles (e.g., as in wind-blown dust), where the flow causes the particles to collide. At each collision there is a probability that a charged species is transferred to a different state on the other particles; this transfer probability is based on a rate constant. The simulations were run with 1000 particles and for durations up to 15 million collisions. 


\section{Results and discussion}

In the experiments that mimic the sand storm, particles were collected at two locations - one location near the bed surface ("bottom"), and the other location farther above the bed ("top"). For each set of collected particles, the particle charge and size distribution were measured. The top cup consistently collected a smaller sample of particles. This result aligns with expectations that the larger heavy particles are not lofted as much as the smaller lighter particles. We now address the effect of an electric field, such as that which is found to occur in dust storms by looking at the difference of the large particle fraction between the bottom and top cups. At an applied potential of $10 \mathrm{kV}$, the difference in large particle fraction was $0.37 \pm 0.7$. At an applied potential of $-10 \mathrm{kV}$, the difference in large particle fraction was $0.27 \pm 0.7$. This result aligns with the idea that large particles charge more positively, and small particles charge more negatively; the result is also corroborated by the charge data collected. In a real dust storm, electric fields build from the charge on the particles themselves. Since larger particles tend to stay lower, the electric field will be oriented with positive on the bottom, and this would have the effect pushing larger particles up and away from the ground.

In the more controlled experiments, we are able to study more carefully the particle-size dependence of the polarity of triboelectric charging. Figure 3 addresses the difference in the particle size distribution of positive and negative particles, as a function of relative humidity. In particular, the Figure shows the difference in the quantity of large particles in the positive and negative samples. All of the values here are positive, indicating that the large particles are more likely to be charged positively rather than negatively. As humidity increases, the difference in the size distribution of the positive and negative samples decreases. We address the physical basis of this experimental result with our Monte Carlo simulations, as described below.

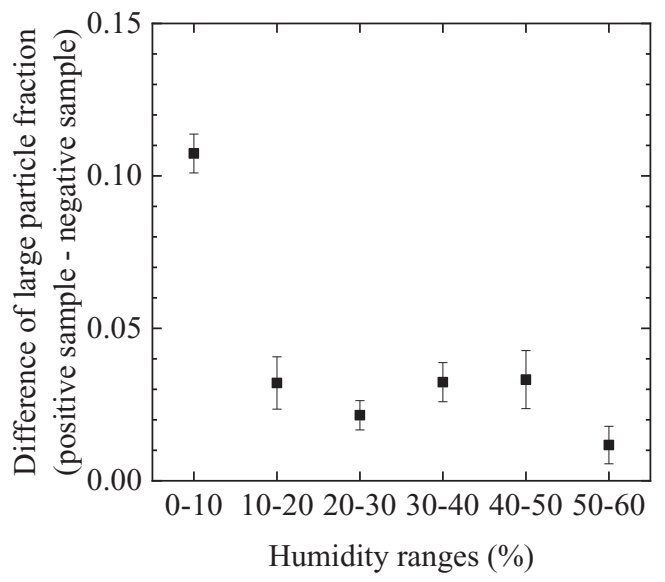

Fig. 3. Difference in large particle fraction between the positive and the negative samples as a function of relative humidity.

We use our Monte Carlo simulations to provide support to our proposed mechanism that humidity leads to a greater build-up of water on the surface of the particles, which could provide a conductive pathway for charged species (electrons or ions) to relax to low energy states on the same particle rather than on the other particle. Figure 4 shows the results of our simulation, in a manner analogous to the experimental results in Figure 3 -i.e., the difference in the quantity of large particles in the positive and negative samples. Again, the results are always positive, and thus the simulations find that our model leads to large particles have a tendency to charge positively. These results also show that as the density of charge carriers in high energy states decreases, the difference plotted in Figure 5 also decreases. Since our proposed mechanism is that the density of charge carriers in high energy states decreases with increasing humidity, the trend from our simulations (Figure 4) matches the trend from our experiments (Figure 3 ).

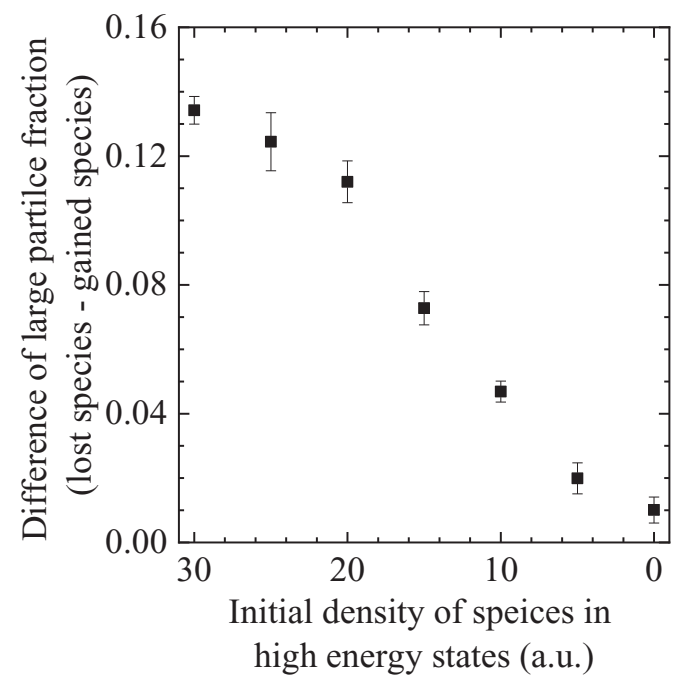

Fig. 4. Difference in large particle fraction between particles that lost charge carriers (positive particles) and particles that gained charge carriers (negative particles) as a function of the initial concentration of charge carriers in high energy states.

Finally, we use our experiments that mimic the sandstorm to address the effects of triboelectric charging on sand transport. Figure 5 shows the quantity of sand collect near the sand bed ("bottom") and at a higher elevation ("top"). The results show that as the electric field increases in magnitude, more sand particles are collected at higher elevation. This result occurs with either polarity of the electric field. Note again that in real dust storms, an electric field develops due to the electrostatic charging of the particles. Thus, our results demonstrate that the electrostatic charging of the particles leads to greater dust transport. 


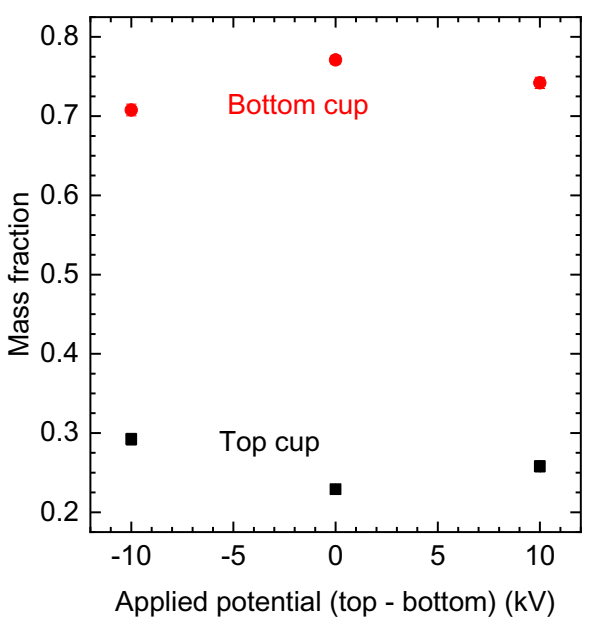

Fig. 5. Dust transported (found in top cup) as a function of applied electric field.

\section{Conclusion}

We have carried out experiments that show that triboelectric charging occurs in wind-blown dust, such that smaller particles tend to charge negatively and larger particles positively. Our theoretical model provides a mechanism for this effect, in terms of non-equilibrium dynamics of charge carriers. The particle-size-dependent polarity of triboelectric charging leads to the formation of electric fields, and the electric fields in turn enhance dust transport when the particles are electrostatically charged.

We would like to acknowledge our colleagues, Dr. Mihai Bilici and Amber Phillips. This material is based upon work supported by the National Science Foundation under grant numbers 1559508, 1604909, and 1206480.

\section{References}

1. W. A. D. Rudge, Proc. R. Soc. A Math. Phys. Eng. Sci. 90, 256-272 (1914)

2. T. Egan, The Worst Hard Time: The Untold Story of Those Who Survived the Great American Dust Bowl, Mariner Books, (2006)

3. J. F. Kok, \& N. O. Renno, J. Phys. Conf. Ser. 142, 012047 (2008)

4. K. R. Rasmussen, J. F. Kok, J. P. Merrison, Planet. Space Sci. 57, 804-808 (2009).

5. E. Williams, N. Nathou, E. Hicks, C. Pontikis, B. Russell, M. Miller, M.J. Bartholomew, Atmos. Res. 91, 292-298 (2009)

6. T.-L. Bo, X.-J. Zheng, Aeolian Res. 8, 39-47 (2013)

7. J. Lowell, A. C. Rose-Innes, Adv. Phys. 29, 9471023, (1980)

8. K. M. Forward, D. J. Lacks, R. M. Sankaran, Ind. Eng. Chem. Res. 48, 2309-2314 (2009)
9. M. A. Bilici, J. R. Toth, R. M. Sankaran, D. J. Lacks, Rev. Sci. Instrum. 85, 103903 (2014)

10.J. R. Toth, A. K. Phillips, S. Rajupet, R. M. Sankaran, D. J. Lacks, Ind. Eng. Chem. Res. 56, 9839-9845 (2017)

11.D. J. Lacks, A. Levandovsky, J. Electrostat. 65, 107-112 (2007) 